\title{
The benefits of cycling
}

\section{Cf \\ alternating \\ drug \\ treatment \\ reduces the \\ emergence of resistance}

To minimize the emergence of antimicrobial resistance, drugs are often used in combination rather than individually, but combination therapy is associated with toxic side effects. As an alternative, the cycling of antibiotics, in which drugs are periodically switched rather than used together, is also suggested to slow down the development of resistance. Using experimental evolution of Staphylococcus aureus, Kim et al. now show that alternating drug treatment reduces the emergence of resistance owing to trade-offs that limit the mutational pathways towards resistance.

The authors evolved $S$. aureus in vitro for 22 days in the presence of trimethoprim (TMP), neomycin (NEO) or ciprofloxacin (CIP), with all possible combinations of two of the drugs or with alternating drugs (in which one of the two antibiotics was used every other day). Interestingly, all cycling treatments

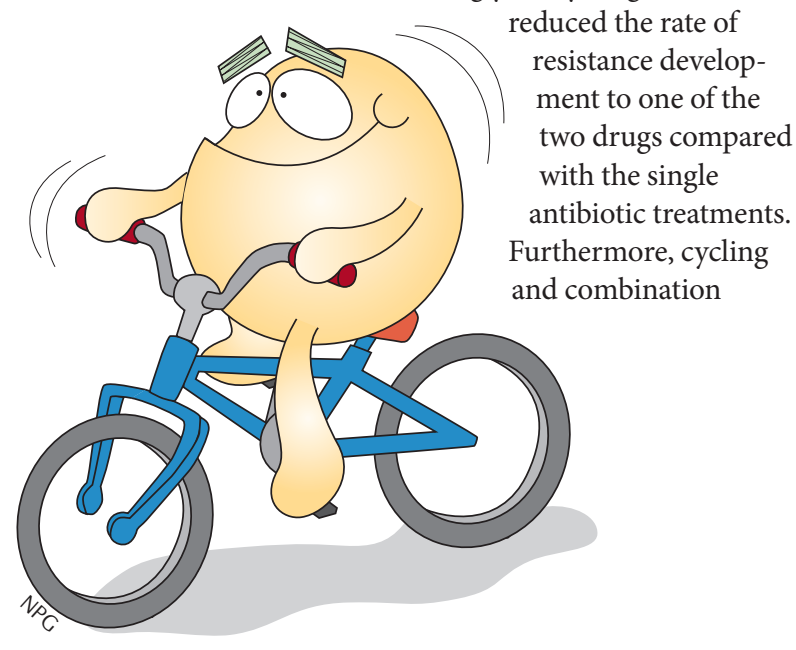

therapy with TMP and CIP had the same effect on the rate of resistance emergence, whereas the cycling of TMP and NEO or NEO and CIP was less effective than the combined treatment. Collectively, these data demonstrate that drug cycling slows down the emergence of resistance compared to using individual drugs, and for some drug pairs it is potentially as effective as using the two drugs simultaneously.

To understand how cycling and combining antibiotics reduces the emergence of resistance, the authors sequenced the genomes of the evolved $S$. aureus populations to identify the resistance mutations associated with each type of therapy. Interestingly, the mutations that arose following drug cycling and combination therapy were similar but they differed from those that were acquired after treatment with single drugs. Both the cycling and combined treatments resulted in several mutations that were not previously associated with resistance, whereas mutations that typically confer resistance to single drugs (mutations in socalled primary resistance genes) were rarely observed. These observations suggest that both types of multidrug treatment constrain the evolution of drug resistance by limiting the acquisition of mutations in primary resistance genes.

Finally, the authors investigated whether collateral antibiotic sensitivity, in which a mutation that provides resistance to one drug confers susceptibility to the other (a trade-off), was responsible for the slower evolution of resistance in bacteria that were treated with alternating drugs. Indeed, collateral sensitivity was frequent in singlestep mutants (which were grown for one generation in the presence of a single antibiotic), especially following TMP treatment; most TMP-resistant mutants were more sensitive to CIP and NEO than wild-type cells, suggesting that trade-offs introduced by drug cycling can limit the mutational pathways that lead to drug resistance.

These data demonstrate that alternating antibiotic treatment can be as effective as combination therapy in limiting the emergence of antimicrobial resistance, but further studies are needed to determine whether drug cycling reduces treatment toxicity. Cláudio Nunes-Alves

ORIGINAL RESEARCH PAPER Kim, S Lieberman, T. D. \& Kishony, R. Alternating antibiotic treatments constrain evolutionary paths to multidrug resistance. Proc. Natl Acad. Sci. USA http://dx.doi.org/10.1073/pnas.1409800111 (2014) 